\title{
COLLISIONALLY INDUCED HYPERFINE-STRUCTURE TRANSITIONS OF OH
}

\author{
SHIH-I CHU \\ Joint Institute for Laboratory Astrophysics, University of Colorado and National Bureau of Standards \\ Received 1975 April 14; revised 1975 November 10
}

\begin{abstract}
The theory of excitation of the internal structures of $\mathrm{OH}$ by electron or heavy-particle impact is formulated within the quantum-mechanical close-coupling approximation, incorporating spin-orbit and hyperfine interactions. In the case of electron impact, explicit Born formulae are derived and the selection rules are discussed for the dipole- and the quadrupole-induced transitions. Numerical results are reported for the electron excitation cross sections of the hyperfine-structure transitions among the lower-lying rotational ladders of the $X^{2} \Pi^{ \pm}{ }_{3 / 2}(v=0)$ and $X^{2} \Pi^{ \pm}{ }_{1 / 2}(v=0)$ states.
\end{abstract}

Subject headings: hyperfine structure - molecular processes - transition probabilities

\section{INTRODUCTION}

Collisional excitation of the internal states of $\mathrm{OH}$ is an interesting and important problem in many astrophysical contexts. In particular, hyperfine-structure excitation cross sections of $\mathrm{OH}$ are critical parameters in the analysis of the population inversion of $\mathrm{OH}$ masers as well as in the calculations of the $\mathrm{OH}$ cooling efficiency in interstellar space. Earlier studies of collisional excitation of $\mathrm{OH}$ did not take into account fine (spin-orbit) and hyperfine interactions (Johnston 1967; Rogers and Barrett 1968; Goss and Field 1968; Doyle and Allison 1972). In this paper, we advance the $\mathrm{OH}$ problem by including the details of the internal structures.

In $\S \mathrm{II}$, the internal wave functions of $\mathrm{OH}$ [between Hund's cases $(a)$ and $(b)$ ] are derived and the scattering problem is formulated in the close-coupling theory. The theory is general enough to be applicable to either electron or heavyparticle impact. In $\S \mathrm{III}$, we present the Born-approximation treatment of the electron impact excitation of $\mathrm{OH}$. Explicit formulae are derived for both dipole- and quadrupole-induced cross sections, and the selection rules for allowed transitions are pointed out. Finally, the excitation cross sections for rotational and hyperfine-structure transitions of the $X^{2} \Pi^{ \pm}{ }_{3 / 2}(v=0)$ and $X^{2} \Pi^{ \pm}{ }_{1 / 2}(v=0)$ states of $\mathrm{OH}$ are calculated and discussed in $\S \mathrm{IV}$.

\section{THEORY}

Consider the excitation of $\mathrm{OH}$ by structureless particles. The Schrödinger equation describing the relative and internal motions is

$$
\left[-\frac{\hbar^{2}}{2 \mu} \nabla_{\boldsymbol{R}}^{2}+\mathscr{H}(\boldsymbol{r}, \boldsymbol{\xi})+V(\boldsymbol{r}, \boldsymbol{R})\right] \Psi=E \Psi,
$$

where $\mu$ is the reduced mass of the colliding system, $\boldsymbol{r}$ is the internuclear-axis vector of $\mathrm{OH}, \xi$ denotes collectively the internal coordinates (electron spin, nuclear spin) of $\mathrm{OH}, \boldsymbol{R}$ is the position vector of the scattered particle measured from the center of mass of $\mathrm{OH}$, and $V$ is the interaction potential, which for the case of atom or electron interacting with a symmetric-top molecule can be represented by the general form

$$
V=\sum_{\lambda=0}^{\infty} \sum_{\mu=-\lambda}^{\lambda} \sum_{\nu=-\lambda}^{\lambda} v_{\lambda \mu}(r, R) D_{\nu \mu}{ }^{(\lambda)}(\alpha \beta \gamma) Y_{\lambda \nu}(\theta, \phi) .
$$

Here $(\alpha \beta \gamma)$ are the Euler angles specifying the orientation of the $\mathrm{OH}$ molecule with respect to the frame of space-fixed (SF) coordinates, $D_{v \mu}{ }^{(\lambda)}$ is the matrix element of the operator of finite rotation, $(\theta, \phi)$ denote the direction of $\boldsymbol{R}$ with respect to the SF frame, and $Y_{\lambda \nu}$ is a spherical harmonic. In equation (1), $\mathscr{H}$ is the internal Hamiltonian of $\mathrm{OH}$, which can be written as

$$
\mathscr{H}=\mathscr{H}_{\text {eN }}+\mathscr{H}_{\text {rso }}+\mathscr{H}_{\text {hf }},
$$

where $\mathscr{H}_{\mathrm{eN}}$ is the nonrelativistic claimed-nuclei Hamiltonian plus the nuclear kinetic energy, $\mathscr{H}_{\mathrm{rso}}$ represents the rigid body rotation and spin-orbit coupling interactions, and $\mathscr{H}_{\mathrm{hf}}$ represents the magnetic hyperfine interactions. In the notation of Dousmanis, Sanders, and Townes (1955), $\mathscr{H}_{\text {rso }}$ and $\mathscr{H}_{\text {hf }}$ are given by

$$
\begin{gathered}
\mathscr{H}_{\mathrm{rso}}=B(\boldsymbol{J}-\boldsymbol{L}-\boldsymbol{S})+A \boldsymbol{L} \cdot \boldsymbol{S}, \\
\mathscr{H}_{\mathrm{hf}}=a \boldsymbol{I} \cdot \boldsymbol{L}+b \boldsymbol{I} \cdot \boldsymbol{S}+c I_{z} S_{z}+\frac{1}{2} d\left(e^{2 i \phi} I^{-} S^{-}+e^{-2 i \phi} I^{+} S^{+}\right)+e\left[e^{i \phi}\left(S^{-} I_{z}+I^{-} S_{z}\right)+e^{-i \phi}\left(S^{+} I_{z}+I^{+} S_{z}\right)\right],
\end{gathered}
$$


where $B$ is the rotational constant, $A$ is the spin-orbit coupling constant, and the quantities $a, b, c, d$, and $e$ are magnetic hyperfine interaction constants. $\boldsymbol{L}, \boldsymbol{S}$, and $\boldsymbol{J}$ are respectively the electron orbital angular momentum, the electron spin angular momentum, and the total angular momentum (exclusive of nuclear spin) and $\boldsymbol{I}$ is the nuclear spin angular momentum. $L^{ \pm}, S^{ \pm}$, and $I^{ \pm}$are the raising and lowering operators for $L, S$, and $I$, respectively. The internuclear axis of $\mathrm{OH}$ is along the body-fixed $z$-direction.

Let $\left|{ }^{2 S+1} \tilde{\Gamma}_{|\Omega|} J I F M_{F}\right\rangle$ be the eigenfunctions of the Hamiltonian $\mathscr{H}$, where $\Gamma=\Sigma, \Pi, \Delta, \ldots$ denotes the electronic state of $\mathrm{OH}, \epsilon(= \pm 1)$ is the state parity, $\boldsymbol{F}(=\boldsymbol{I}+\boldsymbol{J})$ is the total angular momentum and $M_{F}$ is its space-fixed component, and $\Omega$ is the projection of $\boldsymbol{J}$ along the molecular axis. Since $\mathscr{H}_{\mathrm{hf}} \ll \mathscr{H}_{\text {rso }}$, these eigenfunctions can be constructed to a good approximation by

$$
\left.\left.\left.\right|^{2 S+1} \tilde{\Gamma}_{|\Omega|}^{\epsilon} J I F M_{F}\right\rangle\left.\approx \sum_{M} \sum_{M_{I}}\left\langle J M I M_{I} \mid F M_{F}\right\rangle\left|I M_{I}\right\rangle\right|^{2 S+1} \tilde{\Gamma}_{|\Omega|}^{\epsilon} J M\right\rangle
$$

where $\langle\ldots \mid \cdots\rangle$ is the Clebsch-Gordan coefficient (Edmonds 1957), the $\left|I M_{I}\right\rangle$ are nuclear spin eigenfunctions, and the $\left|{ }^{2 S+1} \tilde{\Gamma}_{|\Omega|}^{\epsilon} J M\right\rangle$ are eigenfunctions of $\mathscr{H}_{\mathrm{eN}}+\mathscr{H}_{\mathrm{rso}}$. The matrix elements of $\mathscr{H}_{\text {rso }}$ have been given by Van Vleck (1929) for the basis functions of $\mathscr{H}_{\mathrm{eN}}$ corresponding to the Hund's case $(a)$ coupling scheme. The eigenfunctions of $\mathscr{H}_{\mathrm{eN}}+\mathscr{H}_{\text {rso }}$ can be constructed from the Hund's case $(a)$ basis functions, corresponding to the ${ }^{2} \Pi_{\Omega}$ and ${ }^{2} \Sigma_{\Omega}$ electronic states. For the $\mathrm{OH}$ molecule, the two ground ${ }^{2} \Pi_{|\Omega|}$ states $(|\Omega|=1 / 2,3 / 2)$ are only $140 \mathrm{~cm}^{-1}$ apart whereas the excited ${ }^{2} \Sigma_{1 / 2}$ state lies $32,683 \mathrm{~cm}^{-1}$ above them. Thus to first order, the effect of the $\Sigma$ state on the $\Pi$ states can be ignored and the resulting eigenfunctions of $\mathscr{H}_{\mathrm{eN}}+\mathscr{H}_{\text {rso }}$ (intermediate coupling states) for the two ground ${ }^{2} \Pi$ states are given by (Dousmanis, Sanders, and Townes 1955)

$$
\left.\left.\left|{ }^{2} \Pi_{3 / 2}^{\epsilon} J M\right\rangle=\left.C\left(\epsilon J \frac{3}{2} \mid \frac{1}{2}\right)\right|^{2} \Pi_{1 / 2}^{\epsilon} J M\right\rangle+\left.C\left(\epsilon J \frac{3}{2} \mid \frac{3}{2}\right)\right|^{2} \Pi_{3 / 2}^{\epsilon} J M\right\rangle,
$$

and

$$
\left.\left.\left|{ }^{2} \tilde{\Pi}_{1 / 2} J M\right\rangle=\left.C\left(\epsilon J \frac{1}{2} \mid \frac{3}{2}\right)\right|^{2} \Pi_{3 / 2}^{\epsilon} J M\right\rangle+\left.C\left(\epsilon J \frac{1}{2} \mid \frac{1}{2}\right)\right|^{2} \Pi_{1 / 2}^{\epsilon} J M\right\rangle,
$$

where $\left|{ }^{2} \Pi_{|\Omega|} J M\right\rangle$ on the right-hand side of equation (7) are pure Hund's case (a) wave functions (with state parity $\epsilon)$, and the $C$ coefficients are defined by

$$
C\left(\epsilon J \frac{3}{2} \mid \frac{1}{2}\right)=-C\left(\epsilon J \frac{1}{2} \mid \frac{3}{2}\right)=\left(\frac{X-2+\lambda}{2 X}\right)^{1 / 2},
$$

and

where

$$
C\left(\epsilon J \frac{3}{2} \mid \frac{3}{2}\right)=C\left(\epsilon J \frac{1}{2} \mid \frac{1}{2}\right)=\left(\frac{X+2-\lambda}{2 X}\right)^{1 / 2}
$$

$$
\lambda=A / B=-7.501
$$

(Poynter and Beaudet 1968), and

$$
X=\left[(2 J+1)^{2}+\lambda(\lambda-4)\right]^{1 / 2} .
$$

The structure of the pure Hund's case $(a)$ wave functions $\left|{ }^{2} \Pi_{|\Omega|}^{\epsilon} J M\right\rangle$ deserves further consideration. For simplicity and lucidity, we shall discuss only the electronic-rotational wave functions. (The vibrational wave functions are of little concern in the low-energy scatterings. They can be incorporated easily into the theory if needed.) Because of the $\pm \Lambda$ degeneracy, we are actually concerned here with the scattering between two different electronic states corresponding to $\epsilon= \pm 1$. It is generally more difficult to deal with the multiple surface problem than with the single surface problem.

However, because the $\Lambda$-splitting is very small, one can simply ignore this splitting and treat the problem like a single surface problem (see, for example, Klar 1973). A more rigorous analysis of the $\pm \Lambda$ degeneracy is provided by Green and Zare (1975). They found that the two $\Lambda$ components of each rotational level can be described either as two electronic states or as one electronic state with two rotational modes. The crucial part of their analysis is the separation of rotational and electronic motions and the subsequent ability to choose a representation for these motions which involves a single electronic state. Using their results, our Hund's case $(a)$ wave functions can be decomposed approximately into the product of electronic and rotational wave functions

$$
\left|\Pi_{|\Omega|}^{\epsilon} J M\right\rangle \approx \Phi_{\mathrm{el}}\left({ }^{2} \Pi_{|\Omega|}\right) \cdot|\epsilon J M \Omega\rangle,
$$

where $\Phi_{\mathrm{el}}\left({ }^{2} \Pi_{|\Omega|}\right)$ is the single electronic wave function, and where the rotational wave functions are given by

$$
|\epsilon J M \Omega\rangle=\frac{1}{\sqrt{ } 2}[|J M \bar{\Omega}\rangle+\epsilon|J M-\bar{\Omega}\rangle],
$$


with

$$
|J M \Omega\rangle=\text { symmetric-top wave functions }=\left(\frac{[J]}{8 \pi^{2}}\right)^{1 / 2} D_{M \Omega}^{(J)}(\alpha \beta \gamma),
$$

where

$$
[J] \equiv 2 J+1, \quad \text { and } \quad \bar{\Omega}=|\Omega| .
$$

Consider now the close-coupling formulation of the collisional excitation of the hyperfine-structure levels of the ${ }^{2} \Pi_{|\Omega|}^{\epsilon}$ rigid rotational ladders in the $v=0$ vibrational state. Let $l$ be the angular momentum of the relative motion and $\boldsymbol{J}^{T}$ be the total angular momentum of the collision system defined by

$$
J^{T}=F+l,
$$

where $\boldsymbol{F}$ is the total angular momenturn of $\mathrm{OH}$. (For simplicity we shall ignore the effect of the spin angular momentum of the impact particles. It can be incorporated easily if necessary.) The total wave function $\Psi$ of equation (1) can be expanded in terms of coupled angular basis functions $\Gamma^{J^{T} M^{T}}$ as follows:

$$
\Psi^{j J^{T} M^{T}}=\sum_{\alpha F} \sum_{l} \frac{1}{R} f_{\alpha F l}{ }^{J^{T}}(R) \Gamma_{\alpha F l}{ }^{{ }^{T} M^{T}}
$$

where $\alpha$ are additional quantum numbers needed to specify $F, \alpha=(\epsilon I J \Omega)$, and where

$$
\Gamma_{\alpha F l}^{J^{T} M^{T}}=\sum_{M_{F}, m_{l}}\left\langle F M_{F} l m_{l} \mid J^{T} M^{T}\right\rangle \psi_{\alpha F M_{F}} \cdot Y_{l m_{l}}(\hat{R}),
$$

with $\psi_{\alpha F M_{F}} \equiv\left|{ }^{2} \tilde{\Pi} \epsilon_{|\Omega|} J I F M_{F}\right\rangle$ defined in equation (6). Substituting equation (12) into equation (1), we get a set of coupled differential equations,

$$
\left[\frac{d^{2}}{d R^{2}}-\frac{l(l+1)}{R^{2}}+k_{\alpha F}^{2}\right] f^{J^{T}}{ }_{\alpha F l, \alpha_{0} F_{0} l_{0}}(R)=\frac{2 \mu}{\hbar^{2}} \sum_{\alpha^{\prime} F^{\prime} l^{\prime}} V_{\alpha^{\prime} F^{\prime} l^{\prime}, \alpha F l}^{J^{T}}(R) f^{J^{T}}{ }_{\alpha^{\prime} F^{\prime} l^{\prime}, \alpha F l}(R),
$$

where $k_{\alpha F}^{2}=2 \mu / \hbar^{2}\left[E-\epsilon_{\alpha F}\right]$, with $\epsilon_{\alpha F}$ being the internal energy of $\mathrm{OH}$ in the quantum state $(\alpha F)$. The coupling matrix elements $V^{J^{T}}{ }_{\alpha^{\prime} F^{\prime} l^{\prime}, \alpha F l}(R)$ are given by

$$
V^{J^{T}}{ }_{\alpha^{\prime} F^{\prime} l^{\prime}, \alpha F l}(R)=\iint d \hat{R} d r \Gamma_{\alpha^{T} M^{T} F^{*} l^{\prime}}^{J^{*}} V(\boldsymbol{r}, \boldsymbol{R}) \cdot \Gamma_{\alpha F l} J^{T} M^{T}
$$

which is independent of $M^{T}$. Using the potential given in equation (2), we can evaluate the coupling matrix elements by the methods of Racah algebra to obtain $(\gamma \equiv \alpha F l)$

$$
\begin{aligned}
V_{\gamma^{\prime} \gamma}^{J^{\prime}}(R)= & \sum_{i=1}^{2} \sum_{j=1}^{2} \sum_{\lambda=0}^{\infty} \sum_{\mu=-\lambda}^{\lambda} C_{i}\left(\epsilon^{\prime} J^{\prime} \Omega^{\prime} \mid \Omega_{i}{ }^{\prime}\right) \cdot C_{j}\left(\epsilon J \Omega \mid \Omega_{j}\right) \cdot\left\langle v_{\lambda \mu}(R)\right\rangle_{i j} \cdot \frac{1}{2}\left([J]\left[J^{\prime}\right][F]\left[F^{\prime}\right]\left[l^{\prime}\right][\lambda][l] / 4 \pi\right)^{1 / 2} \\
& \left.\cdot(-1)^{J^{T}-I-\Omega_{i}-\lambda}\left(\begin{array}{lll}
l^{\prime} & \lambda & l \\
0 & 0 & 0
\end{array}\right)\left\{\begin{array}{ccc}
F^{\prime} & \lambda & F \\
J & I & J^{\prime}
\end{array}\right\} \begin{array}{ccc}
l^{\prime} & F^{\prime} & J^{T} \\
F & l & \lambda
\end{array}\right\} \\
& \cdot\left[\left(\begin{array}{ccc}
J^{\prime} & \lambda & J \\
-\Omega_{i}{ }^{\prime} & \mu & \Omega_{j}
\end{array}\right)+\epsilon^{\prime}(-1)^{2 \Omega_{i}{ }^{\prime}}\left(\begin{array}{ccc}
J^{\prime} & \lambda & J \\
\Omega_{i}^{\prime} & \mu & \Omega_{j}
\end{array}\right)+\epsilon\left(\begin{array}{cccc}
J^{\prime} & \lambda & J \\
-\Omega_{i}{ }^{\prime} & \mu & -\Omega_{j}
\end{array}\right)+\epsilon \epsilon^{\prime}(-1)^{2 \Omega_{i}{ }^{\prime}}\left(\begin{array}{ccc}
J^{\prime} & \lambda & J \\
\Omega_{i}^{\prime} & \mu & -\Omega_{j}
\end{array}\right)\right],
\end{aligned}
$$

where $C_{i}$ and $C_{j}$ are the mixing coefficients given in equation (7a) and/or (7b), $(\ldots)$ and $\{\ldots\}$ are respectively the $3 j$ and $6 j$ symbols (Edmonds 1957), and the expectation values $\left\langle v_{\lambda \mu}\right\rangle_{i j}$ are given by

$$
\left\langle v_{\lambda \mu}(R)\right\rangle_{i j}=\iint d \boldsymbol{r} d \xi_{e} \Phi_{\mathrm{el}}^{(i)^{*}}\left({ }^{2} \Pi_{\left|\Omega_{i}\right|}\right) \chi_{\mathrm{vib}}{ }^{(i)}(\boldsymbol{r}) v_{\lambda \mu}(r, R) \Phi_{\mathrm{el}}^{(j)}\left({ }^{2} \Pi_{|\Omega,|}\right) \chi_{\mathrm{vib}}{ }^{(j)}(\boldsymbol{r}),
$$

where the integrations are carried over the electronic coordinates $\xi_{e}$ and the internuclear vector $r$, and $\Phi_{e 1}\left({ }^{2} \Pi_{|\Omega|}\right)$ and $\chi_{\mathrm{vib}}$ are respectively the Hund's case (a) electronic (defined in eq. [8]) and vibrational wave functions.

The coupled differential equations (14) can be solved numerically (cf. Chu and Dalgarno 1975) subject to the following boundary conditions:

$$
f^{J^{T}}{ }_{\alpha^{\prime} F^{\prime} l^{\prime}, \alpha F l}(R=0)=0,
$$


and

$$
\begin{aligned}
& f_{\alpha^{\prime} F^{\prime} l^{\prime}, \alpha F l}^{J^{T}}(R) \underset{R \rightarrow \infty}{\sim} \delta_{\alpha^{\prime} \alpha} \delta_{F^{\prime} F} \delta_{l^{\prime} l}\left[-2 i \sin \left(k_{\alpha F} R-\frac{1}{2} l \pi\right)\right] \\
&+\left(k_{\alpha F} / k_{\alpha^{\prime} F^{\prime}}\right)^{1 / 2} T^{J^{T}}\left(\alpha^{\prime} F^{\prime} l^{\prime} ; \alpha F l\right) \exp \left[i\left(k_{\alpha^{\prime} F^{\prime}} R-l^{\prime} \pi / 2\right)\right],
\end{aligned}
$$

which defines the scattering $T$ matrix. The integral cross section for the $\left(\alpha F \rightarrow \alpha^{\prime} F^{\prime}\right)$ transition, when a summation over $M_{F^{\prime}}$ and an average over $M_{F}$ are taken, is given by

$$
\sigma\left(\alpha F \rightarrow \alpha^{\prime} F^{\prime}\right)=\frac{\pi}{(2 F+1) k_{\alpha F^{2}}} \sum_{J^{T}=0}^{\infty} \sum_{l=\left|J^{T}-F\right|}^{J^{T}+F} \sum_{l^{\prime}=\left|J^{T}-F^{\prime}\right|}^{J^{T}+F^{\prime}}\left|T^{J^{T}}\left(\alpha^{\prime} F^{\prime} l^{\prime}, \alpha F l\right)\right|^{2} .
$$

III. EXCITATION OF THE HYPERFINE-STRUCTURE LEVELS OF OH BY SLOW ELECTRONS:

THE FIRST-ORDER BORN APPROXIMATION

When a molecule is excited by slow electrons, the first-order Born approximation in general gives a fairly good account of the scattering process (Takayanagi 1966; Chang 1970). Perturbation theory has been particularly useful in collisional inelastic studies when the long-range interactions dominate the scattering process. Perturbation theory is also very useful for providing insights into the problems of collisional asymmetries, our main interest in this paper.

The Born formula for the transitions

$$
\left({ }^{2} \tilde{\Pi} \epsilon_{|\Omega|} J I F M_{F} \rightarrow{ }^{2} \tilde{\Pi} \epsilon^{\prime}{ }_{\left|\Omega^{\prime}\right|} J^{\prime} I^{\prime} F^{\prime} M_{F}^{\prime}\right)
$$

at ground vibrational states $\left(v=v^{\prime}=0\right)$ is given by

$\sigma\left({ }^{2} \tilde{\Pi} \epsilon_{|\Omega|} J I F M_{F} \rightarrow{ }^{2} \tilde{\Pi} \epsilon^{\epsilon^{\prime}}{ }_{\left|\Omega^{\prime}\right|} J^{\prime} I^{\prime} F^{\prime} M_{F}{ }^{\prime}\right)=\frac{\tilde{m}^{2}}{4 \pi^{2} \hbar^{4}} \frac{k^{\prime}}{k} \int d \hat{k}^{\prime}\left|\int d \boldsymbol{R} \exp (-i \boldsymbol{q} \cdot \boldsymbol{R})\left\langle{ }^{2} \tilde{\epsilon^{\epsilon^{\prime}}}{ }_{\left|\Omega^{\prime}\right|} J^{\prime} I^{\prime} F^{\prime} M_{F}{ }^{\prime}|V|^{2} \tilde{\Pi} \tilde{\Pi}_{|\Omega|} J I F M_{F}\right\rangle\right|^{2}$,

where $\tilde{m}$ is the reduced mass of the colliding system, $\boldsymbol{k}$ and $\boldsymbol{k}^{\prime}$ are the initial and final wave vectors of the scattered electron, $\hat{k}$ is the unit vector along $\boldsymbol{k}^{\prime}, \boldsymbol{q}=\boldsymbol{k}^{\prime}-\boldsymbol{k}$, and $\boldsymbol{R}$ is the position vector of the electron measured with respect to the center of mass of $\mathrm{OH}$. Using equations (2) and (6)-(10), and the Racah algebra, we obtain for the interaction matrix element,

$$
\begin{aligned}
& \left\langle{ }^{2} \tilde{\Pi}_{\left|\Omega^{\prime}\right|} J^{\prime} I^{\prime} F^{\prime} M_{F}^{\prime}|V|^{2} \tilde{\Pi} \epsilon_{|\Omega|} J I F M_{F}\right\rangle \\
& =\sum_{\lambda \mu \nu} \sum_{i=1}^{2} \sum_{j=1}^{2} C_{i}\left(\epsilon^{\prime} J^{\prime} \Omega^{\prime} \mid \Omega_{i}{ }^{\prime}\right) C_{j}\left(\epsilon J \Omega \mid \Omega_{j}\right)\left\langle v_{\lambda \mu}(R)\right\rangle_{i j} Y_{\lambda \nu}(\theta, \phi)
\end{aligned}
$$

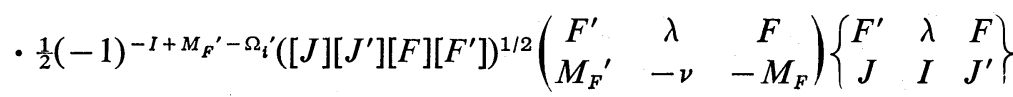

$$
\begin{aligned}
& \cdot\left[\left(\begin{array}{ccc}
J^{\prime} & \lambda & J \\
-\Omega_{i}{ }^{\prime} & \mu & \Omega_{j}
\end{array}\right)+\epsilon^{\prime}(-1)^{2 \Omega_{i}{ }^{\prime}}\left(\begin{array}{ccc}
J^{\prime} & \lambda & J \\
\Omega_{i}{ }^{\prime} & \mu & \Omega_{j}
\end{array}\right)+\epsilon\left(\begin{array}{ccc}
J^{\prime} & \lambda & J \\
-\Omega_{i} & \mu & -\Omega_{j}
\end{array}\right)+\epsilon \epsilon^{\prime}(-1)^{2 \Omega_{i}{ }^{\prime}}\left(\begin{array}{ccc}
J^{\prime} & \lambda & J \\
\Omega_{i}{ }^{\prime} & \mu & -\Omega_{j}
\end{array}\right)\right],
\end{aligned}
$$

where $C_{i}, C_{j}$, and $\left\langle v_{\lambda \mu}\right\rangle_{i j}$ are the same as those given in equation (16). Expanding exp (-iq $\left.\cdot \boldsymbol{R}\right)$ as

$$
\exp (-i \boldsymbol{q} \cdot \boldsymbol{R})=4 \pi \sum_{l m}(-i)^{l} j_{l}(q R) Y_{l m} *(\theta, \phi) Y_{l m}\left(\theta_{q}, \phi_{q}\right),
$$

where $j_{l}$ is the spherical Bessel function, and $\left(\theta_{q}, \phi_{q}\right)$ are the angular coordinates of $\boldsymbol{q}$, and performing the integration over the angular part of $\boldsymbol{R}$ in equation (20), we obtain

$$
\begin{aligned}
& \sigma\left({ }^{2} \tilde{\Pi} \epsilon_{|\Omega|} J I F M_{F} \rightarrow{ }^{2} \tilde{\Pi}{ }^{\epsilon^{\prime}}{ }_{\left|\Omega^{\prime}\right|} J^{\prime} I^{\prime} F^{\prime} M_{F}{ }^{\prime}\right) \\
& =\frac{\tilde{m}^{2}}{\hbar^{4}} \frac{k^{\prime}}{k}\left([J]\left[J^{\prime}\right][F]\left[F^{\prime}\right]\right) \int d \hat{k}^{\prime} \cdot \mid \sum_{\lambda \mu \nu} Y_{\lambda v}\left(\theta_{q}, \phi_{q}\right)(-i)^{\lambda}\left(\begin{array}{ccc}
F^{\prime} & \lambda & F \\
M_{F}^{\prime} & -v & -M_{F}
\end{array}\right)\left\{\begin{array}{ccc}
F^{\prime} & \lambda & F \\
J & I & J^{\prime}
\end{array}\right\} \\
& \cdot \sum_{i j}(-1)^{-I-\Omega_{i} C_{i} C_{j}}\left[\left(\begin{array}{ccc}
J^{\prime} & \lambda & J \\
-\Omega_{i}{ }^{\prime} & \mu & \Omega_{j}
\end{array}\right)+\epsilon^{\prime}(-1)^{2 \Omega_{i}{ }^{\prime}}\left(\begin{array}{ccc}
J^{\prime} & \lambda & J \\
\Omega_{i}{ }^{\prime} & \mu & \Omega_{j}
\end{array}\right)+\epsilon\left(\begin{array}{ccc}
J^{\prime} & \lambda & J \\
-\Omega_{i}^{\prime} & \mu & -\Omega_{j}
\end{array}\right)\right. \\
& \left.+\epsilon \epsilon^{\prime}(-1)^{2 \Omega_{i}}\left(\begin{array}{ccc}
J^{\prime} & \lambda & J \\
\Omega_{i}^{\prime} & \mu & -\Omega_{j}
\end{array}\right)\right]\left.\left[\int R^{2} d R j_{\lambda}(q R)\left\langle v_{\lambda \mu}(R)\right\rangle_{i j}\right]\right|^{2} \text {. }
\end{aligned}
$$


When an average over $M_{F}$ and a summation over $M_{F}{ }^{\prime}$ are taken, the cross section for the transition

$$
\left({ }^{2} \tilde{\Pi}{ }_{|\Omega|} J I F \rightarrow{ }^{2} \tilde{\Pi} \epsilon^{\epsilon^{\prime}}{ }_{\left|\Omega^{\prime}\right|} J^{\prime} I^{\prime} F^{\prime}\right)
$$

becomes

$$
\begin{aligned}
& \sigma\left({ }^{2} \tilde{\Pi}_{|\Omega|} J I F \rightarrow{ }^{2} \tilde{\Pi}{ }^{\epsilon^{\prime}}{ }_{\left|\Omega^{\prime}\right|} J^{\prime} I^{\prime} F^{\prime}\right) \\
& =\frac{\tilde{m}^{2}}{4 \pi \hbar^{4}} \frac{k^{\prime}}{k}[J]\left[J^{\prime}\right]\left[F^{\prime}\right] \cdot \int d \hat{k}^{\prime} \sum_{\lambda}\left\{\begin{array}{ccc}
F^{\prime} & \lambda & F \\
J & I & J^{\prime}
\end{array}\right\}^{2} \\
& \cdot \mid \sum_{\mu} \sum_{i j}(-1)^{-I-\Omega_{i} C_{i}} C_{j}\left[\left(\begin{array}{ccc}
J^{\prime} & \lambda & J \\
-\Omega_{i}{ }^{\prime} & \mu & \Omega_{j}
\end{array}\right)+\epsilon^{\prime}(-1)^{2 \Omega_{i}{ }^{\prime}}\left(\begin{array}{ccc}
J^{\prime} & \lambda & J \\
\Omega_{i}^{\prime} & \mu & \Omega_{j}
\end{array}\right)\right. \\
& \left.\left.+\epsilon\left(\begin{array}{ccc}
J^{\prime} & \lambda & J \\
-\Omega_{i}^{\prime} & \mu & -\Omega_{j}
\end{array}\right)+\epsilon \epsilon^{\prime}(-1)^{2 \Omega_{i}{ }^{\prime}}\left(\begin{array}{ccc}
J^{\prime} & \lambda & J \\
\Omega_{i}{ }^{\prime} & \mu & -\Omega_{j}
\end{array}\right)\right] \cdot \mid \int_{0}^{\infty} R^{2} d R j_{\lambda}(q R)\left\langle v_{\lambda \mu}(R)\right\rangle_{i j}\right]\left.\right|^{2} .
\end{aligned}
$$

The radial integral in equation (24) can be evaluated analytically by substituting the asymptotic form of the electron-molecule long-range potential,

$$
\left\langle v_{\lambda \mu}(R)\right\rangle_{i j}=-\left\langle M_{\lambda \mu}\right\rangle_{i j} / R^{\lambda+1}
$$

where the leading nonvanishing terms of $\left\langle M_{\lambda \mu}\right\rangle_{i j}$ for the case of $\mathrm{OH}$ are given by

$$
\begin{aligned}
& M_{10}=\left(\frac{4}{3} \pi\right)^{1 / 2}\left\langle Q_{z}^{(1)}\right\rangle_{i j}, \\
& M_{20}=\left(\frac{4}{5} \pi\right)^{1 / 2}\left\langle Q_{z z}{ }^{(2)}\right\rangle_{i j}, \\
& M_{22}=M_{2,-2}=-\left(\frac{4 \pi}{30}\right)^{1 / 2}\left\langle Q_{z z}{ }^{(2)}-Q_{y y}{ }^{(2)}\right\rangle_{i j},
\end{aligned}
$$

where $\langle Q\rangle_{i j}$ is the electronic transition moment of the operator $Q$ between Hund's case $(a)$ states $|i\rangle$ and $|j\rangle$, $Q_{z}{ }^{(1)}=\hat{D}$ is the dipole moment operator, and $Q_{k k}{ }^{(2)}(k=x, y, z)$ are diagonal quadrupole moment tensor components. (The body frame $z$-axis is chosen to be along the internuclear axis $\boldsymbol{r}$, i.e., $\boldsymbol{z} \| \boldsymbol{r}$.)

Substituting equation (25) into equation (24) and using the transformation

$$
\int d \hat{k}^{\prime} \rightarrow 2 \pi \int_{\left|k-k^{\prime}\right|}^{k+k^{\prime}} \frac{q d q}{k k^{\prime}}
$$

the final expression for the cross section can be reduced to the form

$$
\sigma\left({ }^{2} \widetilde{\Pi}_{|\Omega|} J I F \rightarrow{ }^{2} \widetilde{\Pi}^{\epsilon^{\prime}}{ }_{\left|\Omega^{\prime}\right|} J^{\prime} I^{\prime} F^{\prime}\right)=\sum_{\lambda \mu} \sigma_{\lambda \mu}\left({ }^{2} \widetilde{\Pi}_{|\Omega|}^{\epsilon} J I F \rightarrow{ }^{2} \tilde{\Pi} \epsilon_{\left|\Omega^{\prime}\right|} J^{\prime} I^{\prime} F^{\prime}\right),
$$

where $\sigma_{\lambda \mu}$ represents the contribution from each term of the interaction $v_{\lambda \mu}$. The first few leading terms of $\sigma_{\lambda \mu}$ are

$$
\begin{aligned}
& \sigma_{10}\left({ }^{2} \tilde{\Pi}_{|\Omega|}{ }_{|\Omega|} J I F \rightarrow{ }^{2} \tilde{\Pi} \epsilon^{\epsilon^{\prime}}{ }_{\left|\Omega^{\prime}\right|} J^{\prime} I^{\prime} F^{\prime}\right) \\
& =\frac{8 \pi \tilde{m}^{2}}{3 \hbar^{4} k^{2}}\left([J]\left[J^{\prime}\right]\left[F^{\prime}\right]\right) \cdot \frac{1}{2}\left[1-\epsilon \epsilon^{\prime}(-1)^{J-J^{\prime}}\right] \\
& \cdot\left\{\begin{array}{ccc}
F^{\prime} & 1 & F \\
J & I & J^{\prime}
\end{array}\right\}^{2}\left|\sum_{i, j=1}^{2}(-1)^{I-\Omega_{i}{ }^{\prime}} C_{i}\left(\epsilon^{\prime} J^{\prime} \Omega^{\prime} \mid \Omega_{i}{ }^{\prime}\right) C_{j}\left(\epsilon J \Omega \mid \Omega_{j}\right)\langle D\rangle_{i j}\left(\begin{array}{ccc}
J^{\prime} & 1 & J \\
-\Omega_{i}{ }^{\prime} & 0 & \Omega_{j}
\end{array}\right) \delta_{\Omega_{i}{ }^{\prime} \Omega_{j}}\right|{ }^{2} \ln \left|\frac{k+k^{\prime}}{k-k^{\prime}}\right|, \\
& \sigma_{20}\left({ }^{2} \widetilde{\Pi}_{|\Omega|}^{\epsilon} J I F \rightarrow{ }^{2} \widetilde{\Pi} \epsilon^{\epsilon^{\prime}}{ }_{\left|\Omega^{\prime}\right|} J^{\prime} I^{\prime} F^{\prime}\right) \\
& =\frac{16 \pi \tilde{m}^{2}}{45 \hbar^{4}}\left(\frac{k^{\prime}}{k}\right)\left([J]\left[J^{\prime}\right]\left[F^{\prime}\right]\right) \cdot \frac{1}{2}\left[1+\epsilon \epsilon^{\prime}(-1)^{J-J^{\prime}}\right]\left\{\begin{array}{ccc}
F^{\prime} & 2 & F \\
J & I & J^{\prime}
\end{array}\right\}^{2} \\
& \cdot\left|\sum_{i, j=1}^{2}(-1)^{I-\Omega_{i}{ }^{\prime}} C_{i}\left(\epsilon^{\prime} J^{\prime} \Omega^{\prime} \mid \Omega_{i}{ }^{\prime}\right) C_{j}\left(\epsilon J \Omega \mid \Omega_{j}\right)\left\langle Q_{z z}{ }^{(2)}\right\rangle_{i j}\left(\begin{array}{ccc}
J^{\prime} & 2 & J \\
-\Omega_{i}{ }^{\prime} & 0 & \Omega_{j}
\end{array}\right) \delta_{\Omega_{\Omega^{\prime}} \Omega_{j}}\right|^{2},
\end{aligned}
$$


and

$$
\begin{aligned}
\left(\sigma_{22}+\sigma_{2,-2}\right) & \left({ }^{2} \tilde{\Pi} \epsilon_{|\Omega|} J I F \rightarrow{ }^{2} \tilde{\Pi}_{\left|\Omega^{\prime}\right|}^{\epsilon^{\prime}} J^{\prime} I^{\prime} F^{\prime}\right) \\
= & \frac{8 \pi \tilde{m}^{2}}{135 \hbar^{4}}\left(\frac{k^{\prime}}{k}\right) \cdot\left([J]\left[J^{\prime}\right]\left[F^{\prime}\right]\right) \cdot \frac{1}{2}\left[1+\epsilon \epsilon^{\prime}(-1)^{\left.J-J^{\prime}\right]} \cdot\left\{\begin{array}{ccc}
F^{\prime} & 2 & F \\
J & I & J^{\prime}
\end{array}\right\}^{2}\right. \\
& \cdot\left|\sum_{i, j=1}^{2}(-1)^{I-\Omega_{i}^{\prime}} C_{i}\left(\epsilon^{\prime} J^{\prime} \Omega^{\prime} \mid \Omega_{i}{ }^{\prime}\right) C_{j}\left(\epsilon J \Omega \mid \Omega_{j}\right)\left\langle Q_{x x}{ }^{(2)}-Q_{y y}{ }^{(2)}\right\rangle_{i j}\left(\begin{array}{ccc}
J^{\prime} & 2 & J \\
-\Omega_{i}{ }^{\prime} & 2 & -\Omega_{j}
\end{array}\right) \cdot\left(1-\delta_{\left.\Omega_{i}^{\prime} \Omega_{j}\right)}\right)\right|^{2} \cdot
\end{aligned}
$$

The allowed transitions induced by dipole and quadrupole interactions are determined by the $6 j$-symbol $\left\{\begin{array}{ccc}F^{\prime} & \lambda & F \\ J & I & J^{\prime}\end{array}\right\}$ as well as by the factor $\frac{1}{2}\left[1 \pm \epsilon \epsilon^{\prime}(-1)^{J-J^{\prime}}\right]$. The selection rules are summarized in Table 1. Note that the dipole and the quadrupole interactions cause different types of transitions. The presence of the mixing coefficients $C_{i}$ and $C_{j}$ in equations (29)-(31) is essential to account for the cross transitions between the ${ }^{2} \Pi^{ \pm}{ }_{3 / 2}$ and ${ }^{2} \Pi^{ \pm}{ }_{1 / 2}$ states. Were the $\mathrm{OH}$ molecule to belong to a pure Hund's case $(a)$ state, both $v_{10}$ and $v_{20}$ could cause transitions only within either the ${ }^{2} \Pi_{3 / 2}$ or the ${ }^{2} \Pi_{1 / 2}$ ladders. [Note the presence of $\delta_{\Omega_{i} \Omega_{j}}$ in equations (29) and (30).) The $v_{22}$ and $v_{2,-2}$ interactions, which possess the angular $\phi$-dependence, would then become the leading contributors to the cross transitions [note the presence of the $\left(1-\delta_{\Omega_{i}^{\prime} \Omega_{j}}\right)$ factor in eq. (31).]

\section{RESULTS AND DISCUSSION}

In this section, we shall present some of the calculated results for electron impact excitations using the Born formulae (29)-(31). The only molecular parameters that are required are $\left\langle Q_{\lambda \mu}\right\rangle_{i j}$. Because of the presence of the factor $\delta_{\Omega_{i}^{\prime} \Omega_{j}}$ in equations (29) and (30) and the factor $\left(1-\delta_{\Omega_{i}{ }^{\prime} \Omega_{j}}\right)$ in equation (31), only three electronic transition multipole moments are relevant and we have adopted the following values for the dipole and quadrupole transition moments:

$$
\begin{aligned}
\left\langle{ }^{2} \Pi_{3 / 2}\left|\hat{Q}_{z}^{(1)}\right|{ }^{2} \Pi_{3 / 2}\right\rangle & =\left\langle{ }^{2} \Pi_{1 / 2}\left|\hat{Q}_{z}^{(1)}\right|^{2} \Pi_{1 / 2}\right\rangle=D=1.668 \text { debye (Meerts and Dymanus 1973), } \\
\left\langle{ }^{2} \Pi_{3 / 2}\left|\hat{Q}_{z z}{ }^{(1)}\right|{ }^{2} \Pi_{3 / 2}\right\rangle & =\left\langle{ }^{2} \Pi_{1 / 2}\left|\hat{Q}_{z z}{ }^{(2)}\right|^{2} \Pi_{1 / 2}\right\rangle=Q=1.35 \text { electron-bohrs }{ }^{2} \text { (Chu et al. 1974), }
\end{aligned}
$$

and

$$
\left|\left\langle^{2} \Pi_{3 / 2}\left|Q_{x x}{ }^{(2)}-Q_{y y}{ }^{(2)}\right|^{2} \Pi_{1 / 2}\right\rangle\right|=\left|\left\langle^{2} \Pi_{1 / 2}\left|Q_{x x}{ }^{(2)}-Q_{y y}{ }^{(2)}\right|^{2} \Pi_{3 / 2}\right\rangle\right|=\left|Q_{\perp}\right| \approx 2.62 \text { electron-bohrs }^{2},
$$

where $Q_{\perp}$ is estimated from the molecular properties calculated by Chu, Yoshimine, and Liu (1974) and the expectation values of $\left(y^{2}-x^{2}\right)$ calculated by Gwin et al. (1973).

The expressions (29) and (30) can now be rewritten more explicitly $\left(I=I^{\prime}=\frac{1}{2}\right.$, and $\left.v=v^{\prime}=0\right)$,

$$
\begin{aligned}
\sigma_{10}\left({ }^{2} \tilde{\Pi}_{|\Omega|}^{\epsilon} J I F \rightarrow{ }^{2} \tilde{\Pi}_{\left|\Omega^{\prime}\right|}^{\epsilon^{\prime}} J^{\prime} I^{\prime} F^{\prime}\right)= & \frac{8 \pi \tilde{m}^{2} D^{2}}{3 \hbar^{4} k^{2}}\left([J]\left[J^{\prime}\right]\left[F^{\prime}\right]\right) \cdot \frac{1}{2}\left[1-\epsilon \epsilon^{\prime}(-1)^{\left.J-J^{\prime}\right]}\right. \\
& \cdot\left\{\begin{array}{rrr}
F^{\prime} & 1 & F \\
J & \frac{1}{2} & J^{\prime}
\end{array}\right\}^{2} \cdot \ln \left|\begin{array}{l}
k+k^{\prime} \\
k-k^{\prime}
\end{array}\right| \cdot Z\left(1, \Omega, \Omega^{\prime}\right),
\end{aligned}
$$

\begin{tabular}{|c|c|c|}
\hline Type of Interaction & $\Delta J=J^{\prime}-J$ & Does Parity $(\epsilon)$ Change? \\
\hline $\begin{array}{l}\text { Dipole................ } \\
\text { Quadrupole.......... }\end{array}$ & $\begin{array}{l}\text { Even } \\
\text { Odd } \\
\text { Even } \\
\text { Odd }\end{array}$ & $\begin{array}{l}\text { Yes } \\
\text { No } \\
\text { No } \\
\text { Yes }\end{array}$ \\
\hline \multicolumn{3}{|c|}{$\begin{array}{l}\text { Triangle selection rules: } \\
\qquad|F-\lambda| \leq F^{\prime} \leq F+\lambda \\
|J-\lambda| \leq J^{\prime} \leq J+\lambda \\
|J-I| \leq F \leq J+I\left(I=\frac{1}{2}\right) \\
\left|J^{\prime}-I^{\prime}\right| \leq F^{\prime} \leq J^{\prime}+I^{\prime}\left(I^{\prime}=\frac{1}{2}\right)\end{array}$} \\
\hline
\end{tabular}

and

$\sigma_{20}\left({ }^{2} \tilde{\Pi}_{|\Omega|}^{\epsilon} J F \rightarrow{ }^{2} \tilde{\Pi}_{\left|\Omega^{\prime}\right|} J^{\prime} I^{\prime} F^{\prime}\right)=\frac{16 \pi \tilde{m}^{2} Q^{2}}{45 \hbar^{4}}\left(\frac{k^{\prime}}{k}\right)\left([J]\left[J^{\prime}\right]\left[F^{\prime}\right]\right) \cdot \frac{1}{2}\left[1+\epsilon \epsilon^{\prime}(-1)^{\left.J-J^{\prime}\right]}\left\{\begin{array}{ccc}F^{\prime} & 2 & F \\ J & \frac{1}{2} & J^{\prime}\end{array}\right\}^{2} \cdot Z\left(2, \Omega, \Omega^{\prime}\right)\right.$

TABLE 1

Selection Rules for the Dipole $(\lambda=1)$ AND Quadrupole $(\lambda=2)$ INDUCED HyPERFINE-STRUCTURE TRANSITIONS OF OH 
and

$$
\begin{aligned}
& \left(\sigma_{22}+\sigma_{2,-2}\right)\left({ }^{2} \tilde{\Pi}{ }_{|\Omega|} J I F \rightarrow{ }^{2} \tilde{\Pi} \epsilon^{\prime}{ }_{\left|\Omega^{\prime}\right|} J^{\prime} I^{\prime} F^{\prime}\right) \\
& \quad=\frac{8 \pi \tilde{m}^{2} Q_{\perp}{ }^{2}}{135 \hbar^{4}}\left(\frac{k^{\prime}}{k}\right) \cdot\left([J]\left[J^{\prime}\right]\left[F^{\prime}\right]\right) \cdot \frac{1}{2}\left[1+\epsilon \epsilon^{\prime}(-1)^{J-J^{\prime}}\right] \cdot\left\{\begin{array}{ccc}
F^{\prime} & 2 & F \\
J & \frac{1}{2} & J^{\prime}
\end{array}\right\}^{2} \cdot Y\left(2, \Omega, \Omega^{\prime}\right),
\end{aligned}
$$

where $Z\left(\mu, \Omega, \Omega^{\prime}\right)$ and $Y\left(2, \Omega, \Omega^{\prime}\right)$ are given by

$$
\begin{aligned}
Z\left(\mu, \frac{3}{2}, \frac{3}{2}\right)= & \left(\frac{X(J)-2+\lambda}{2 X(J)}\right)^{1 / 2}\left(\frac{X\left(J^{\prime}\right)-2+\lambda}{2 X\left(J^{\prime}\right)}\right)^{1 / 2}\left(\begin{array}{ccc}
J^{\prime} & \mu & J \\
-\frac{1}{2} & 0 & \frac{1}{2}
\end{array}\right) \\
& -\left(\frac{X(J)+2-\lambda}{2 X(J)}\right)^{1 / 2}\left(\frac{X\left(J^{\prime}\right)+2-\lambda}{2 X\left(J^{\prime}\right)}\right)^{1 / 2}\left(\left.\begin{array}{ccc}
J^{\prime} & \mu & J \\
-\frac{3}{2} & 0 & \frac{3}{2}
\end{array}\right|^{2},\right. \\
Z\left(\mu, \frac{1}{2}, \frac{1}{2}\right)= & \mid\left(\frac{X(J)-2+\lambda}{2 X(J)}\right)^{1 / 2}\left(\frac{X\left(J^{\prime}\right)-2+\lambda}{2 X\left(J^{\prime}\right)}\right)^{1 / 2}\left(\begin{array}{ccc}
J^{\prime} & \mu & J \\
-\frac{3}{2} & 0 & \frac{3}{2}
\end{array}\right) \\
& -\left.\left(\frac{X(J)+2-\lambda}{2 X(J)}\right)^{1 / 2}\left(\frac{X\left(J^{\prime}\right)+2-\lambda}{2 X\left(J^{\prime}\right)}\right)^{1 / 2}\left(\begin{array}{ccc}
J^{\prime} & \mu & J \\
-\frac{1}{2} & 0 & \frac{1}{2}
\end{array}\right)\right|^{2}, \\
Z\left(\mu, \frac{3}{2}, \frac{1}{2}\right)= & \mid\left(\frac{X(J)-2+\lambda}{2 X(J)}\right)^{1 / 2}\left(\frac{X\left(J^{\prime}\right)+2-\lambda}{2 X\left(J^{\prime}\right)}\right)^{1 / 2}\left(\begin{array}{ccc}
J^{\prime} & \mu & J \\
-\frac{1}{2} & 0 & \frac{1}{2}
\end{array}\right) \\
& +\left.\left(\frac{X(J)+2-\lambda}{2 X(J)}\right)^{1 / 2}\left(\frac{X\left(J^{\prime}\right)-2+\lambda}{2 X\left(J^{\prime}\right)}\right)^{1 / 2}\left(\begin{array}{ccc}
J^{\prime} & \mu & J \\
-\frac{3}{2} & 0 & \frac{3}{2}
\end{array}\right)\right|^{2}, \\
Z\left(\mu, \frac{1}{2}, \frac{3}{2}\right)= & \mid\left(\frac{X(J)+2-\lambda}{2 X(J)}\right)^{1 / 2}\left(\frac{X\left(J^{\prime}\right)-2+\lambda}{2 X\left(J^{\prime}\right)}\right)^{1 / 2}\left(\begin{array}{ccc}
J^{\prime} & \mu & J \\
-\frac{1}{2} & 0 & \frac{1}{2}
\end{array}\right) \\
& +\left.\left(\frac{X(J)-2+\lambda}{2 X(J)}\right)^{1 / 2}\left(\frac{X\left(J^{\prime}\right)+2-\lambda}{2 X\left(J^{\prime}\right)}\right)^{1 / 2}\left(\begin{array}{ccc}
J^{\prime} & \mu & J \\
-\frac{1}{2} & 0 & \frac{3}{2}
\end{array}\right)\right|^{2},
\end{aligned}
$$

and

and

$$
\begin{aligned}
Y\left(2, \frac{3}{2}, \frac{3}{2}\right)= & Y\left(2, \frac{1}{2}, \frac{1}{2}\right) \\
= & \mid\left(\frac{X(J)+2-\lambda}{2 X(J)}\right)^{1 / 2}\left(\frac{X\left(J^{\prime}\right)-2+\lambda}{2 X\left(J^{\prime}\right)}\right)^{1 / 2}\left(\begin{array}{ccc}
J^{\prime} & 2 & J \\
-\frac{1}{2} & 2 & -\frac{3}{2}
\end{array}\right) \\
& -\left.\left(\frac{X(J)-2+\lambda}{2 X(J)}\right)^{1 / 2}\left(\frac{X\left(J^{\prime}\right)+2-\lambda}{2 X\left(J^{\prime}\right)}\right)^{1 / 2}\left(\begin{array}{ccc}
J^{\prime} & 2 & J \\
-\frac{3}{2} & 2 & -\frac{1}{2}
\end{array}\right)\right|^{2},
\end{aligned}
$$

$$
\begin{aligned}
Y\left(2, \frac{3}{2}, \frac{1}{2}\right)= & Y\left(2, \frac{1}{2}, \frac{3}{2}\right) \\
= & \mid\left(\frac{X(J)+2-\lambda}{2 X(J)}\right)^{1 / 2}\left(\frac{X\left(J^{\prime}\right)+2-\lambda}{2 X\left(J^{\prime}\right)}\right)^{1 / 2}\left(\begin{array}{ccc}
J^{\prime} & 2 & J \\
-\frac{1}{2} & 2 & -\frac{3}{2}
\end{array}\right) \\
& +\left.\left(\frac{X(J)-2+\lambda}{2 X(J)}\right)^{1 / 2}\left(\frac{X\left(J^{\prime}\right)-2+\lambda}{2 X\left(J^{\prime}\right)}\right)^{1 / 2}\left(\begin{array}{ccc}
J^{\prime} & 2 & J \\
-\frac{3}{2} & 2 & -\frac{1}{2}
\end{array}\right)\right|^{2},
\end{aligned}
$$

where

$$
X(J) \equiv\left[(2 J+1)^{2}+\lambda(\lambda-4)\right]^{1 / 2} \text {, and } \lambda=-7.051 \text { and } \mu=1 \text { or } 2 .
$$

The dipole transition expression (eq. [32]) can be alternatively written in terms of the Einstein $A$-coefficient,

$$
\sigma_{10}\left({ }^{2} \tilde{\Pi}_{|\Omega|} J I F \rightarrow{ }^{2} \tilde{\Pi}_{\left|\Omega^{\prime}\right|} J^{\prime} I^{\prime} F^{\prime}\right)=\frac{2 \pi \tilde{m}^{2} c^{3}}{h^{3} \nu^{3} k^{2}} \cdot \frac{1}{2}\left[1-\epsilon \epsilon^{\prime}(-1)^{J-J^{\prime}}\right] \cdot A\left(\Omega J F \rightarrow \Omega^{\prime} J^{\prime} F^{\prime}\right) \cdot \ln \left|\frac{k+k^{\prime}}{k-k^{\prime}}\right|,
$$

where $\nu$ is the radiation transition frequency, $c$ is the velocity of light, and the $A$-coefficient is defined by

$$
A\left(\Omega J F \rightarrow \Omega^{\prime} J^{\prime} F^{\prime}\right)=\frac{64 \pi^{4}}{3 h c^{3}} \cdot D^{2} \cdot \nu^{3} \cdot\left[F^{\prime}\right]\left[J^{\prime}\right][J] \cdot\left\{\begin{array}{ccc}
F^{\prime} & 1 & F \\
J & \frac{1}{2} & J^{\prime}
\end{array}\right\}^{2} \cdot Z\left(1, \Omega, \Omega^{\prime}\right) .
$$

The Einsten $A$-coefficients have been calculated by Burdyuzha and Varshalovich (1973) for various radio and infrared transitions of $\mathrm{OH}$. Thus equation (36) is a useful representation for calculating the dipolar collisional transition probabilities.

Figure 1 shows the energy-dependent excitation cross sections of various hyperfine-structure transitions within each rotational state induced by the dipole interaction. The differences among the hyperfine-structure transitions 


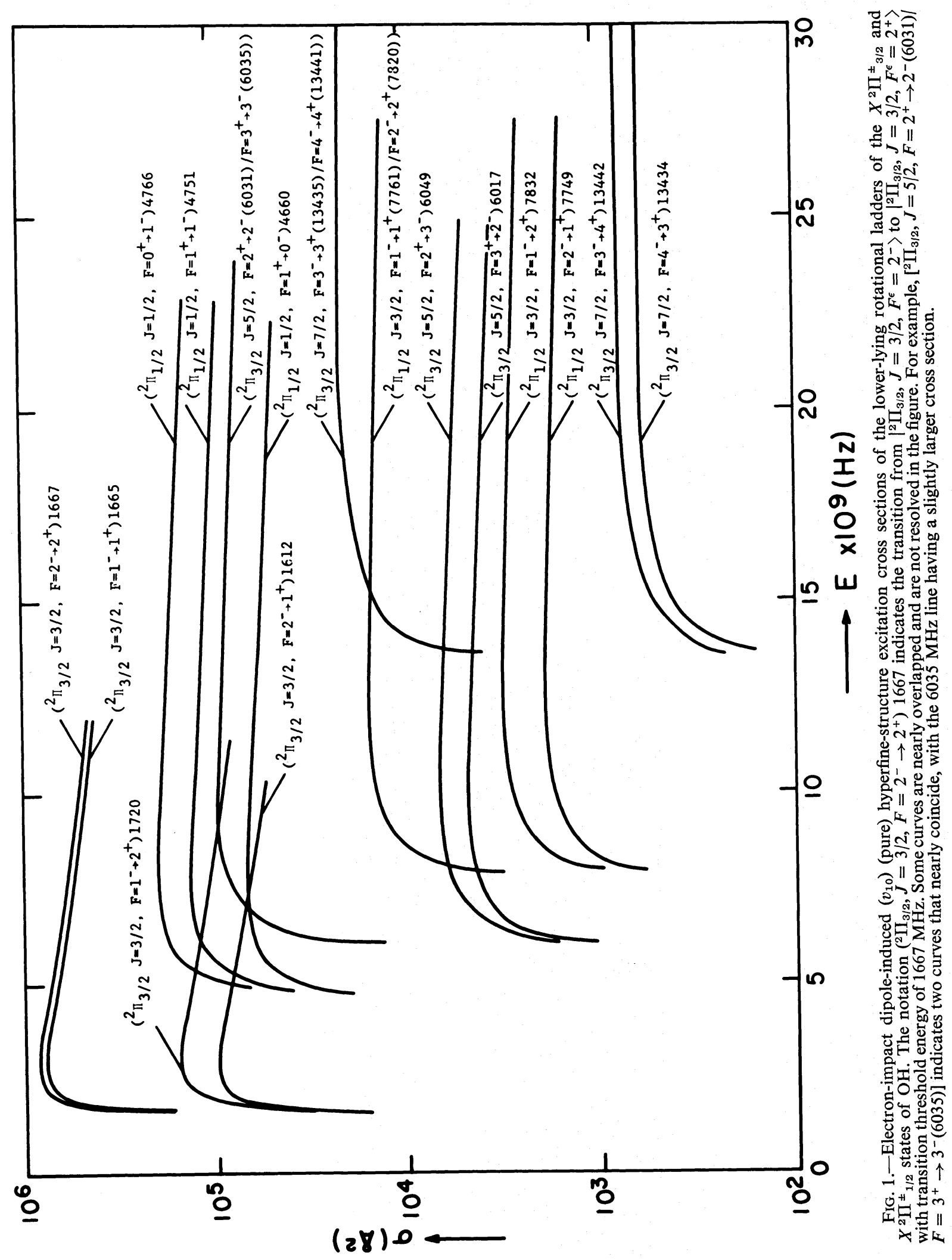




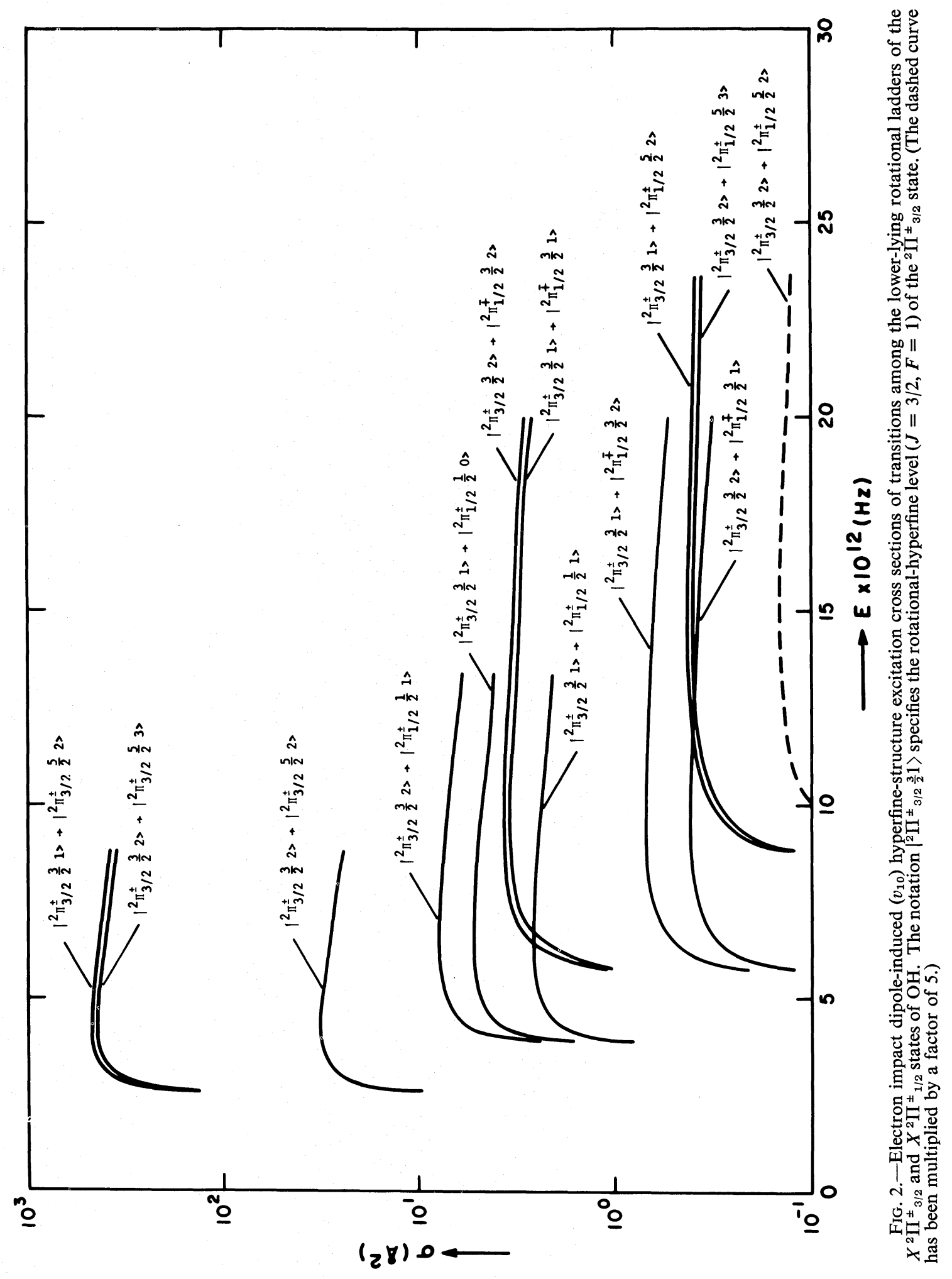




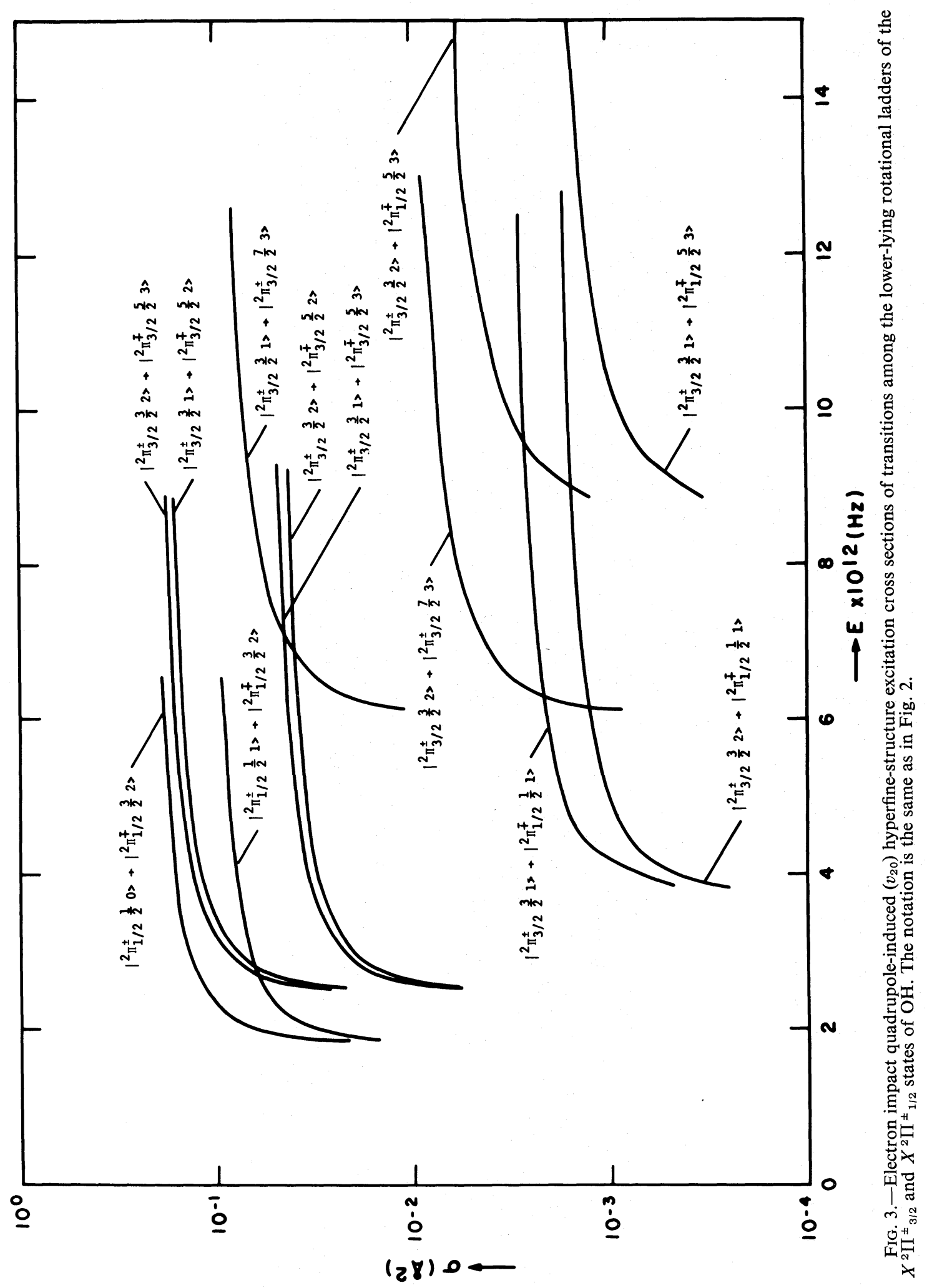




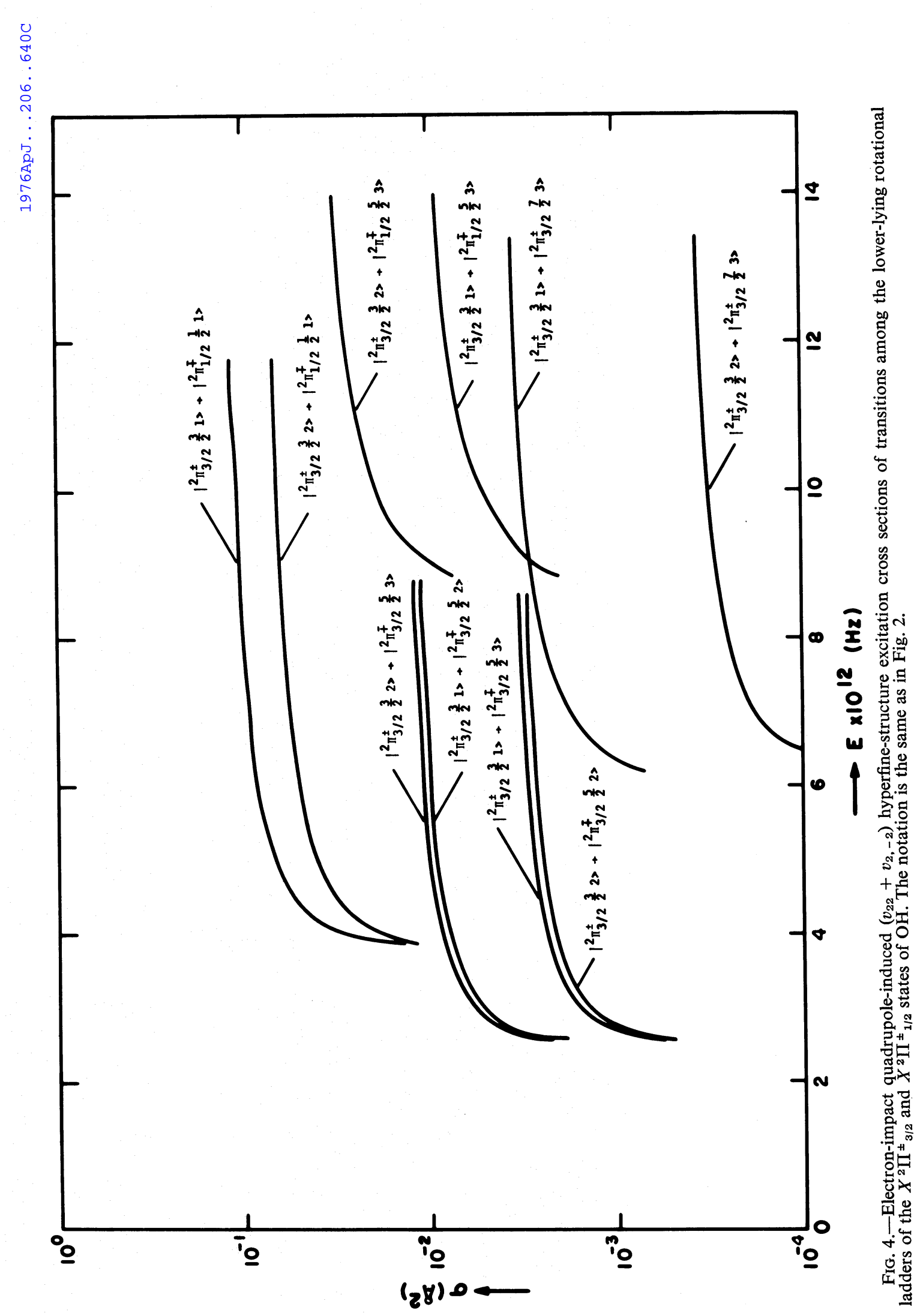


(belonging to the same rotational state) are caused mainly by the differences in their $6 j$ symbols $\left\{\begin{array}{ccc}F^{\prime} & 1 & F \\ J & \frac{1}{2} & J\end{array}\right\}$. Thus the transition probabilities with $\Delta F\left(=F^{\prime}-F\right)=0$ are usually larger than those with $\Delta F \neq 0$. It is noted that the ground rotational state $\left.\left.\right|^{2} \Pi^{ \pm}{ }_{3 / 2}, J=3 / 2\right\rangle$ possesses the largest collisional transition probabilities. The hyperfine-structure excitation cross sections for excited rotational states decrease quite rapidly with increasing rotational quantum numbers $J$.

Figure 2 depicts the dipole-induced hyperfine-structure transition cross sections between different rotational states. Generally, these cross sections are two to three orders of magnitude smaller than those hyperfine-structure transitions within the same rotational state (cf. Fig. 1). Figure 2 shows that for those hyperfine-structure transitions [connected by the same initial $(\Omega, J)$ and the same final $\left(\Omega^{\prime}, J^{\prime}\right)$ rotational states] which do not change state parity, the $\Delta F \neq 0$ transitions have larger excitation cross sections than those of the $\Delta F=0$ transitions. The reverse is true, however, for transitions which change the state parity. These asymmetric collisional pumping phenomena may be very important to the interpretation of the anomalous population of the energy levels of interstellar $\mathrm{OH}$ (see ter Haar and Pelling 1974 for a review of astrophysical masers).

In addition, the significant difference in the transition probabilities between the $\Delta F=0$ and $\Delta F \neq 0$ transitions strongly argues for the necessity of a detailed account the hyperfine structures of the rotational ladders of $\mathrm{OH}$ in the calculation of the molecular cooling problem, namely, collisional excitation of the rotational levels of $\mathrm{OH}$ followed by radiative escape. A recent study by Dalgarno et al. (1974) suggests that the $\mathrm{OH}$ molecule may be important to the cooling of interstellar clouds. These authors found that hydrogen-containing diatomic molecules with large rotational constants may have enhanced cooling rates, with respect to ${ }^{12} \mathrm{CO}$, under certain physical conditions of the clouds.

Figure 3 depicts the quadrupole-induced hyperfine-structure transition cross sections $\sigma_{20}$ between different rotational states, and Figure 4 shows the corresponding behavior for $\sigma_{22}+\sigma_{2,-2}$. Note the shifting of the curves of $\left(\sigma_{22}+\sigma_{2,-2}\right)$ with respect to the corresponding ones of $\sigma_{20}$. For example, the four curves belonging to the rotational transition $\left.\left|{ }^{2} \Pi^{ \pm}{ }_{3 / 2}, J=3 / 2\right\rangle \rightarrow||^{2} \Pi^{\mp}{ }_{3 / 2}, J=5 / 2\right\rangle$ in Figure 3 are depressed down to an order of magnitude in Figure 4. On the other hand, the two curves belonging to the rotational transition $\left|{ }^{2} \Pi^{ \pm}{ }_{3 / 2}, J=3 / 2\right\rangle \rightarrow$ $\left|{ }^{2} \Pi^{\mp}{ }_{1 / 2}, J=1 / 2\right\rangle$ in Figure 3 are enhanced more than an order of magnitude in Figure 4. The presence of the $v_{22}$ and $v_{2,-2}$ interactions thus has the important consequence of causing collisional asymmetry in quadrupole transitions. Similar collisional asymmetry behavior has been observed in the rotational scattering of ${ }^{1} \Pi$-state molecules like $\mathrm{Na}_{2}$ (Bergmann and Demtröder 1972) and $\mathrm{Li}_{2}$ (Ottinger and Poppe 1971) by rare gases. In all cases it was found that for transitions with odd $\Delta J$ values the cross sections $\sigma(+|\Delta J|)$ and $\sigma(-|\Delta J|)$ differ considerably, and it was necessary to propose the $\phi$-dependent potential (like $v_{22}$ and $v_{2,-2}$ ) to explain these observable discrepancies.

The cross sections for quadrupole-induced transitions usually are several orders of magnitude smaller than those of dipole-induced transitions. However, because the dipole and quadrupole transitions obey different selection rules (cf. Table 1), they do not mix together and both are leading contributors to the hyperfine-structure transition cross sections.

The Born approximation, while providing a reasonable method for dealing with electron impact scattering, is not reliable for heavy particles, whose scattering is generally dominated by short-range interactions. A refined method such as the close-coupling theory described in § II may be required.

This research was supported by the National Science Foundation under grant No. MPS 72-05026 A02. The author is indebted to Professor Alexander Dalgarno for his reading and suggestions on the manuscript and to Professor William Reinhardt for his comments.

\section{REFERENCES}

Bergmann, K., and Demtröder, W. 1972, J. Phys. B: Atom. Molec. Phys., 5, 2098.

Burdyuzha, V. V., and Varshalovich, D. A. 1973, Soviet Astr.-AJ, 16, 980.

Chang, E. S. 1970, Phys. Rev. A, 2, 1403.

Chu, S. I., and Dalgarno, A. 1975, Proc. Roy. Soc. A, 342, 191.

Chu, S. I., Yoshimine, M., and Liu, B. 1974, J. Chem. Phys., 61, 5389.

Dalgarno, A., de Jong, T., Oppenheimer, M., and Black, J. H. 1974, Ap. J. (Letters), 192, L37.

Dousmanis, G. C., Sanders, T. M., and Townes, C. H. 1955, Phys. Rev., 100, 1735.

Doyle, H. T., and Allison, A. C. 1972, Atomic Physics 3, ed. S. J. Smith and G. K. Walters (New York: Plenum), p. 62. Edmonds, A. R. 1957, Angular Momentum in Quantum Mechanics (Princeton: Princeton University Press).

Goss, W. M., and Field, G. B. 1968, Ap. J., 151, 177.
Green, S., and Zare, R. N. 1975, Chem. Phys., 7, 62.

Gwinn, W. D., Turner, B. E., Goss, W. M., and Blackman, G. L. 1973, Ap. J., 179, 789.

Johnston, I. D. 1967, Ap. J., 150, 33.

Klar, H. 1973, J. Phys. B: Atom. Molec. Phys., 6, 2139.

Meerts, W. L., and Dymanus, A. 1973, Chem. Phys. Letters, $23,45$.

Ottinger, Ch., and Poppe, D. 1971, Chem. Phys. Letters, 8, 513.

Poynter, R. L., and Beaudet, R. A. 1968, Phys. Rev. Letters, 21, 305 .

Rogers, A. E. E., and Barrett, A. H. 1968, Ap. J., 151, 163.

Takayanagi, K. 1966, J. Phys. Soc. Japan, $21,507$.

ter Haar, D., and Pelling, M. A. 1974, Rept. Progr. Phys., 37, 481 .

Van Vleck, J. H. 1929, Phys. Rev., 33, 467.

SHIH-I CHU: Joint Institute for Laboratory Astrophysics, University of Colorado, Boulder, CO 80302 